\title{
Internal levels of plant growth regulators during in vitro culture of wild cherry (Prunus avium L.)
}

\author{
P. Label ${ }^{1}$, D. Cornu 1 , B. Sotta ${ }^{2}$ and E. Miginiac ${ }^{2}$ \\ 1 INRA, Station d'Amélioration des Arbres Forestiers, Ardon, 45160 Olivet, and \\ 2 Université P.-et-M.-Curie, Laboratoire de Physiologie du Développement des Plantes, 4, pl. \\ Jussieu, T53-E5, 75252 Paris Cedex 05, France
}

\section{Introduction}

In vitro micropropagation of wild cherry is presently one of the main commercial ways to clonally propagate this species (Cornu and Boulay, 1986). In order to extend this technique to a large number of clones, it seems necessary to improve our knowledge of the behavior of the explants during the in vitro culture. Since plant growth regulators (PGR) play an important role in this technique (Margara, 1961), our attention was drawn to the effect of exogenous PGR on hormonal levels in the explants.

\section{Materials and Methods}

Wild cherry explants were cultured according to the procedure described by Riffaud and Cornu (1981). The micropropagation technique can be schematically divided into 3 stages: the multiplication stage, when axillary bud growth is promoted by an almost equal amount of indole3-butyric acid (IBA, $4.9 \mu \mathrm{M}$ ) and benzyladenine $(\mathrm{BA}, 4.4 \mu \mathrm{M})$ in the culture medium; the elongation phase which was not studied; and the rooting phase, in which IBA $(4.9 \mu \mathrm{M})$ alone promoted root formation.
Hormonal measurements were made during the multiplication and the rooting stages. For each measurement, 48 explants were divided into 3 parts: the apical part, including the apex sensu stricto and the youngest leaves inserted in the short internodes of the stem tip; the middle part of the explants, bearing the oldest leaves at the axis, whose axillary buds started to grow during multiplication treatment; and the basal part including the portion of the stem inserted into the culture medium, where roots were formed during the rooting stage.

For each series, explants were collected 0,1 , 2, 4 and $8 \mathrm{~d}$ after their transfer into fresh medium. Frozen samples were lyophilized and ground up with a ball mill. Analytical measurements were made following the procedure reported elsewhere (Label et al., 1989). Techniques used were methanolic extraction, HPLC purification and fractionation, and immunological measurement (ELISA), (Leroux et al., 1985; Maldiney et al., 1986; Sotta et al., 1987; Label and Sotta, 1988). ELISA measurements were repeated 5 times. Mean values are given.

\section{Results}

Morphological development

Under standard multiplication conditions (Fig. 1A, B and C), axillary buds located in 
the middle part of the explants started to grow on $d 4$ and, after 4 wk of culture, the multiplication rate was 3 . When IBA was omitted from this culture medium (Fig. 1B and $\mathrm{C}$ ), no multiplication was observable; moreover, $82 \%$ of the explants were necrotic at the 4 th $w \mathrm{k}$. When BA was omitted from the standard multiplication medium (Fig. 1A), about $65 \%$ of the explants were rooted 4 wh after subculture.

Under standard rooting conditions (Fig. 1D), first root primordia were histologically observable on d 5 and, after 3 wk of culture, $80 \%$ of the explants had at least one root. When IBA was omitted from this culture medium (Fig. 1D), $3 \%$ of the explants were rooted at the $3 \mathrm{rd}$ wk.

\section{Hormonal measurements}

Endogenous hormonal levels are presented on the same unit scale in each figure (with and without exogenous PGR). This was done to point out some specta-
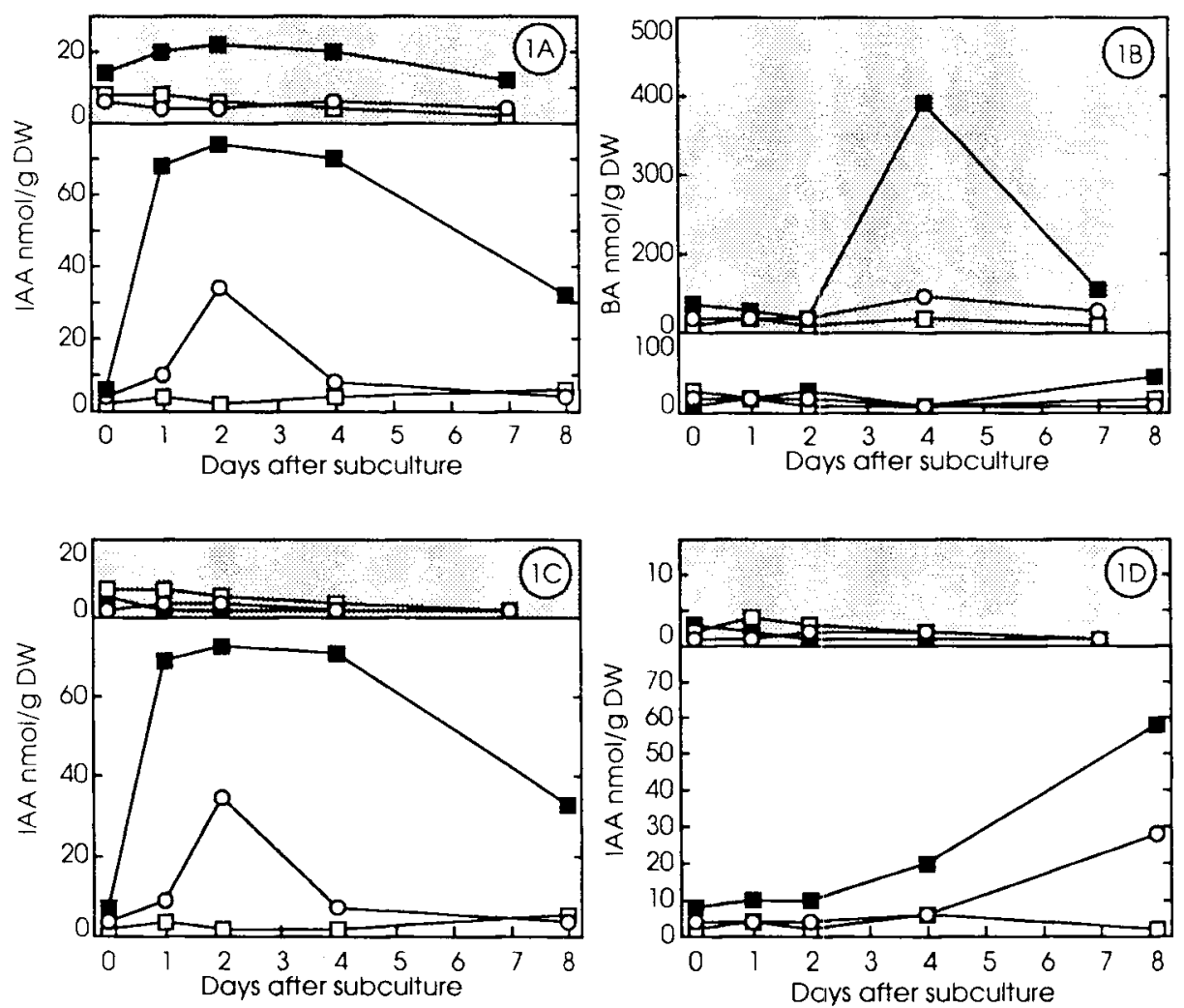

Fig. 1. Internal levels of $I A A$ and $B A$ during the first $8 \mathrm{~d}$ of culture. A: effect of exogenous $B A$ on endogenous IAA content during multiplication; $\mathbf{B}$ : effect of exogenous IBA on internal BA content during multiplication; C: effect of exogenous IBA on endogenous IAA content during multiplication; $D$ : effect of exogenous IBA on endogenous IAA content during rooting. $D$ : apical part; $O$ : middle part; $\mathbf{a}$ : basal part; without exogenous PGR in the culture medium; $\mathbf{0}$ : with exogenous PGR in the culture medium (standard conditions). 
cular differences between hormonal levels in the explants according to treatment. For instance, in Fig. $1 C$ and $D$, without IBA in the culture medium, IAA levels were very low (grey background), but the apico-basal distribution of this hormone in the explants was, nevertheless, significant. Results are presented in $\mathrm{nmol} \cdot \mathrm{g}^{-1} \mathrm{DW}$; to approxi-

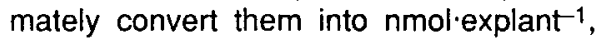
measurements given in the apical, middle and basal part will be multiplied by 3,2 and 1 , respectively.

\section{Discussion and Conclusion}

A relationship between IBA and endogenous IAA can be evoked. In each experiment, when explants were cultured with IBA (multiplication, rooting) in the culture medium, internal IAA was basoapically distributed in the explants and IAA levels were 20-30 times higher than in explants cultured without IBA, where IAA is apico-basally distributed. Epstein and Lavée (1986) reported a transformation of IBA into IAA during in vitro culture of Vitis vinifera and Olea europea. The chemical pathway could be a $\beta$-oxidation of the butyric side chain, but the biochemical mechanism of this process remains unknown.

From the experiments run in the presence and in the absence of $B A$ in the culture medium (Fig. 1B), we postulate that IBA might control the penetration of $B A$ into the explants. We still have to investigate this point, and experiments using radiolabeled IBA and $B A$ would be of great interest in this perspective.

The last result was not illustrated because of its strong clarity: when BA was present in the culture medium (with or without (BA), no natural cytokinins could be detected in the explants, whereas each time BA was removed from the culture medium, natural cytokinins could be quantified by the ELISA technique. Thus, BA had a clear depressive effect on endogenous natural cytokinin metabolism. Although BA metabolism is well known (Letham and Palni, 1983), results on the effect of BA on endogenous cytokinin metabolism have never been published. In the future, the intensity of this depressive effect and the biological activity of this synthetic PGR should be explored.

\section{Acknowledgments}

The technical assistance of P. Capelli and R. Camelin is gratefully acknowledged. This work was partly supported by a grant (no. 4473) from INRA.

\section{References}

Cornu D. \& Boulay M. (1986) La multiplication végétative. Techniques horticoles et culture in vitro. Rev. For. Fr. 38, 60-68

Epstein E. \& Lavée S. (1986) Conversion of indole-3-butyric acid to indole-3-acetic acid by cuttings of gravepine (Vitis vinifera) and olive (Olea europea). Plant Cell Physiol. 253, 697703

Label P. \& Sotta B. (1988) An ELISA test for BA measurement: application to wild cherry culture in vitro. Plant Cell Tissue Organ Culture 1, 155158

Label P., Maldiney R., Sossountzov L., Cornu D. \& Miginiac E. (1989) Endogenous levels of abscisic acid, indole-3-acetic acid and benzyladenine during in vitro bud growth induction of wild cherry (Prunus avium L.). Plant Growth Regul. 8, 325-333

Leroux B., Maldiney R., Miginiac E., Sossountzov L. \& Sotta B. (1985) Comparative quantitation of abscisic acid in plant extracts by gas-liquid chromatography and an enzymelinked immunosorbent assay using the avidin-biotin system. Planta 166, 524-529

Letham D.S. \& Palni L.M.S. (1983) The biosynthesis and metabolism of cytokinins. Annu. Rev. Plant Physiol. 34, 163-197

Maldiney R., Leroux B., Sabbagh I., Sotta B., Sossountzov L. \& Miginiac E. (1986) A bio- 
tin-avidin-based enzyme immunoassay to quantify three phytohormones: auxin, abscisic acid and zeatin riboside. J. Immunol. Methods 90, 151-158

Margara J. (1961) Les corrélations d'inhibition. Ann. Physiol. Vég. 8, 55-69

Riffaud J.L. \& Cornu D. (1981) Utilisation de la culture in vitro pour la multiplication de meri- siers adultes (Prunus avium L.) sélectionnés en forêt. Agronomie 1, 633-640

Sotta B., Pilate G., Pelese F., Sabbagh I., Bonnet M. \& Maldiney R. (1987) An avidin-biotin solid phase ELISA for femtomole isopentenyladenine and isopentenyladenosine measurements in HPLC-purified plant extracts. Plant Physiol. 84, 571-573 